\title{
Li2CaGeO4 - Wide Band Gap Semiconductor: First Principles Investigation of the Structural, Electronic, Optical and Elastic Properties
}

\author{
Saadi Berri \\ Laboratory for Developing New Materials and Their Characterizations, University \\ of Setif 1, Algeria \\ Department of Physics, Faculty of Science, University of M'sila, Algeria
}

\begin{abstract}
The electronic structure and some of its derived properties of $\mathrm{Li}_{2} \mathrm{CaGeO}_{4}$ compound have been investigated. The calculations have been performed using the fullpotential linearized augmented plane wave plus local orbitals method and ultra-soft pseudo-potentials . The optimized lattice parameters are found to be ingood accord with experiment. Features such as bulk modulus and its pressure derivative, electronic band structure and density of states are reported. The elastic anisotropy of the crystal is discussed and visualized. Moreover, the optical properties reveal that $\mathrm{Li}_{2} \mathrm{CaGeO}_{4}$ compound are suitable candidates for optoelectronic devices in the visible and ultraviolet (UV) regions.
\end{abstract}

Keywords: Quaternary diamond; Elastic modulus; Optic-electronic properties; Ab-initio calculations.

Email : berrisaadi12@yahoo.fr

: saadi.berri@univ-msila.dz

\section{INTRODUCTION}

Quaternary diamond-like materials of general formula $\mathrm{AB}_{2} \mathrm{CX}_{4}$ have attracted a great deal of attention followed by the discovery of exotic properties such as tunneling semiconductors [1], photovoltaic's [2], spintronics [3], non-linear optics [4], thermoelectrics [5], magnetics [6] and solar cells [7]. As an example, quaternary diamond- 
like semiconductors $\mathrm{AgCd}_{2-x} \mathrm{Mn}_{x} \mathrm{GaS}_{4}$ and $\mathrm{Ag}_{2} \mathrm{CdSnS}_{4}$ were reported to be as the photoactive centers [8], and optoelectronic application [9].

Wide bandgap semiconductors(WBS) are electronic materials in which the energy of the band-to-band electronic transitions exceeds approximately $3 \mathrm{eV}$. These materials have different kinds of chemical bonds and of crystal lattice structures, but the electronic and optical processes taking place in them have a great deal in common. Diamond, silicon carbide, gallium phosphide, cadmium sulfide, and some other related compounds of the $\mathrm{A}^{\mathrm{II}} \mathrm{B}^{\mathrm{VI}}$ type occupy a special place among the widegap semiconductors[10].

At the same time, research on other semiconductors, and especially wide bandgap semiconductors have allowed to fabricate various power devices reliable and performant enough to design high e ciency level converters in order to match applications requirements. Among these wide bandgap materials, $\mathrm{SiC}$ is the most advanced from a techno- logical point of view: Schottky diodes are already ommercially available since 2001, JFET and MOSFET will be versy soon[11]. However, only a limited data have been reported on the fundamental properties of $\mathrm{Li}_{2} \mathrm{CaGeO}_{4}$ compound. This has motivated us to investigate on the structural, electronic, elastic and optical properties of this material by performing the band structure calculations using the full-potential augmented plane-wave (FP-LAPW) and the pseudopotential plane wave (PP-PW) methods. The remaining of the paper is organized as follows: the theoretical background is described in Section 2. Results are presented and discussed in Section 3. A summary of the results is given in Section 4.

\section{COMPUTATIONAL METHODS}

The atomic structure of $\mathrm{Li}_{2} \mathrm{CaGeO}_{4}$ compound is known to crystallize in a tetragonal lattice, which has space group I-42m (121), where Li atom occupy $4 \mathrm{~d}(0,0.5,0.25)$, Ca at $2 \mathrm{~b}(0,0,0.5)$ sites, Ge on $2 \mathrm{a}(0,0,0)$ and $\mathrm{O}$ at $8 \mathrm{i}(0.189,0.189,0.142$ of tetragonal unit cell [12]. The crystal structures of $\mathrm{Li}_{2} \mathrm{CaGeO}_{4}$ alloy is shown in Fig. 1.

The present computations are performed through the FP-LAPW method using DFT as implemented in WIEN2K code [13]. In the study of structural properties, the exchange correlation energy is treated within the GGA method [14]. We have used $l \max =10$ for angular momentum expansion and $R_{\mathrm{MT}} K_{\max }=8$ as a plane wave cut-off with $3000 \mathrm{k}$ points for tetragonal phase. The radii $R_{\mathrm{MT}}$ of the muffin tins (MT) are chosen to be approximately proportional to the corresponding ionic radii. The energy between successive iterations is 
converged to $0.0001 \mathrm{Ry}$ and forces are minimized to $1 \mathrm{mRy} \mathrm{Bohr}^{-1}$. A dense k-mesh with $10000 \mathrm{k}$-points was used in the first Brillouin zone to calculate the optical properties.

We have also used the ultra-soft pseudo-potentials of the Vanderbilt-type [14] and the GGA according to GGA-sol approach [15] was already used to calculate structural, electronic, elastic and optiacl properties of $\mathrm{Li}_{2} \mathrm{CaGeO}_{4}$. A computer program CASTEP (Cambridge Serial Total Energy Package) [16]. The kinetic cut-off energy for the plane wave expansion is taken to be $500 \mathrm{eV}$ for all cases being considered here. The special $\mathrm{k}$ point sampling for the integration of first Brillouin zone has been employed by using the Monkhorst-Pack method with $14 \times 14 \times 18$ k-points for tetragonal phase. Based on the Broyden Fletcher Goldfarb Shenno (BFGS) [17] minimization technique, the system reached the ground state via self consistent calculation when the total energy is stable to within $5 \times 10^{-6} \mathrm{eV} /$ atom the force is less than $10^{-2} \mathrm{eV} / \mathrm{A}$. A dense k-mesh with $24 \times 24 \times 29 \mathrm{k}$ points was used in the first Brillouin zone to calculate the optical properties.

The optical properties of matter can be described by the complex dielectric function $\varepsilon(\omega)$, which represents the linear response of the system to an external electromagnetic field with a small wave vector. It can be expressed as

$$
\varepsilon(\omega)=\varepsilon_{1}(\omega)+i \varepsilon_{2}(\omega)
$$

The investigated compound crystallizes in the tetragonal symmetry. This symmetry group has two dominant components of the dielectric tensor. These dielectric functions are $\varepsilon_{2}{ }^{\mathrm{zz}}(\omega)$ and $\varepsilon_{2}{ }^{\mathrm{xx}}(\omega)$, corresponding to the electric field direction parallel and perpendicular to the crystallographic $\mathrm{c}$ axis. The calculation of $\varepsilon_{2}(\omega)$ requires the precise values of energy eigen values and electron wave functions. These are natural outputs of a band structure calculation.

The frequency dependent complex dielectric tensor $\varepsilon_{2}(\omega)$ components are calculated by using the following mathematical expressions [18]:

$$
\begin{aligned}
& \varepsilon_{2}^{z z}(\omega)=\frac{12}{m \omega^{2}} \int_{B Z} \sum \frac{\left|p_{n n^{\prime}}^{z z}(k)\right|^{2}}{\nabla \omega_{n n^{\prime}}(k)} d S_{k} \\
& \varepsilon_{2}^{x x}(\omega)=\frac{12}{m \omega^{2}} \int_{B Z} \sum \frac{\left|p_{n n^{\prime}}^{z z}(k)\right|^{2}+\left|p_{n n^{\prime}}^{x x}(k)\right|^{2}}{\nabla \omega_{n n^{\prime}}(k)} d S_{k}
\end{aligned}
$$


In the above relations, $\mathrm{e}^{2}=1 / \mathrm{m}=1$ and $\mathrm{h}=1$ (written in atomic unit) where " $\omega$ " is the photon frequency of energy “ $\hbar \omega$ ", and $p_{n n^{\prime}}^{x, y}(k)$ represent the $\mathrm{x} / \mathrm{y}$-components of the dipolar matrix elements between initial $\left\langle n k /(\right.$ Bra $)$ states and final $\left./ n^{\prime} k\right\rangle$ (ket) states.

$\omega_{n n^{\prime}}(k)=$ Difference in band energies $=$ En $(\mathrm{k})-E_{n^{\prime}}(k)($ energy eigen values $)$, and the last term

$S_{k}=$ constant surface energy $=\mathrm{k} ; \quad \omega_{n n^{\prime}}(k)=\omega$. The real part $\varepsilon_{1}(w)$ of the dielectric function $\varepsilon_{2}(w)$ is derived from $\varepsilon_{2}(w)$ by the Kramers-Kronig transformation.

$$
\varepsilon_{1}(\omega)=1+\frac{2}{\pi} M \int_{0}^{\infty} \frac{\omega^{\prime} \varepsilon_{2}\left(\omega^{\prime}\right)}{\omega^{\prime 2}-\omega^{2}} d \omega^{\prime}
$$

where $M$ implies the principle value of the integral.

The optical constants such as refractive index $n(w)$ and the extinction coefficient $k(w)$, are calculated intermsof the real and the imaginary parts of the complex dielectric function as follows [19, 20].

$$
\begin{aligned}
& n=\frac{\left(\varepsilon_{1}+\left(\varepsilon_{1}^{2}+\varepsilon_{2}^{2}\right)^{\frac{1}{2}}\right)^{\frac{1}{2}}}{\sqrt{2}} \\
& k=\frac{\left(-\varepsilon_{1}+\left(\varepsilon_{1}^{2}+\varepsilon_{2}^{2}\right)^{\frac{1}{2}}\right)^{\frac{1}{2}}}{\sqrt{2}}
\end{aligned}
$$

\section{RESULTS AND DISCUSSION}

The experimental lattice parameters has been optimized using Birch-Murnaghan's [21] equation of state by fitting energy vs cell volume. Figure 1 presents the structural optimization curves obtained by using the FP-LAPW method. This allowed the determination of the equilibrium lattice constants $a(\AA)$ and $c(\AA)$ and bulk modulus $B(\mathrm{GPa})$ and its first pressure derivative $B^{\prime}$. The resulting structural parameters for both methods being considered in the present work are listed in Table 1. The optimal lattice parameters $a(\AA)$ and $c(\AA) \quad$ obtained by this procedure accords well with the experimental values reported in Ref. [12]. Based on the experimental data, the equilibrium lattice constants for 
$\mathrm{Li}_{2} \mathrm{CaGeO}_{4}$ are best described by PP-PW, compared with the FP-LAPW method. Nevertheless, both used methods overestimated the lattice constant with respect to experiment.

Table.1 Lattice constant $a(\AA), \quad c(\AA)$, bulk modulus $B$ (in GPa), and first-pressure derivative of bulk modulus $B$ ' for $\mathrm{Li}_{2} \mathrm{CaGeO}_{4}$.

\begin{tabular}{|l|l|l|l|l|}
\hline & $\mathrm{a}(\AA)$ & $\mathrm{c}(\AA)$ & $B(\mathrm{GPa})$ & $B^{\prime}$ \\
\hline FP-LAPW & 5.258 & 6.745 & 120.446 & 4.232 \\
\hline PP-PW & 5.155 & 6.988 & 68.852 & - \\
\hline EXP[12] & $5.141 \pm .002$ & $6.595 \pm 0.002$ & - & - \\
\hline
\end{tabular}

To study the mechanical stability and the stiffness of the the newly $\mathrm{Li}_{2} \mathrm{CaGeO}_{4}$ compound, we have calculated the single-crystal elastic constants $C_{i j}$ using the finite elastic strain method [22]. A tetragonal crystal has six different elastic constants, namely: $\mathrm{C}_{11}$, $\mathrm{C}_{12}, \mathrm{C}_{13}, \mathrm{C}_{33}, \mathrm{C}_{44}$ and $\mathrm{C}_{66}$. Among these, $C_{66}$ is dependent as $C_{66}=\left(C_{11}-C_{12}\right) / 2$. These constants must be satisfying thefollowing well-known mechanical stability conditions [23, 24]:

$$
\mathrm{C}_{44}>0, \quad \mathrm{C}_{11}-\mathrm{C}_{12}>, \quad\left(\mathrm{C}_{11}+2 \mathrm{C}_{12}\right) \mathrm{C}_{33}-2 \mathrm{C}^{2}{ }_{13}>0
$$

The calculated constants $\mathrm{C}_{\mathrm{ij}}$ allow us to obtain the macroscopic mechanical parameters of these compounds, namely their bulk $(B)$ and shear $(G)$ moduli can be computed with the aid of the main approximation Voigt-Reuss-Hill [25], and compressibility $(\beta=1 / B)$.

The resulting calculated constants $\mathrm{C}_{\mathrm{ij}}$ for $\mathrm{Li}_{2} \mathrm{CaGeO}_{4}$ compound being considered in the present work are listed in Table 2. For the tetragonal system, the Voigt approximation [26] of the bulk modulus $B_{V}$ and shear modulus $G_{V}$ are:

$$
B_{V}=\frac{2}{9}\left(C_{11}+C_{12}+\frac{C_{33}}{2}+2 C_{13}\right)
$$

and 


$$
G_{V}=\frac{1}{30}\left(C_{11}+C_{12}+2 C_{33}-4 C_{13}+12 C_{44}+12 C_{66}\right.
$$

the Reuss approximation[27] of the bulk modulus and shear modulus are :

$$
B_{R}=\frac{\left(C_{11}+C_{12}\right) C_{33}-2 C_{13}^{2}}{C_{11}+C_{12}+2 C_{33}-4 C_{13}}
$$

and

$$
G_{R}=\frac{5}{2}\left\{\frac{\left[\left(C_{11}+C_{12}\right) C_{33}-2 C_{13}^{2}\right] C_{44} C_{66}}{3 B_{v} C_{44} C_{66}+\left[\left(C_{11}+C_{12}\right) C_{33}-2 C_{13}^{2}\right]\left(C_{44}+C_{66}\right)}\right\}
$$

Table 2: The calculated elastic constants $\mathrm{C}_{\mathrm{ij}}(\mathrm{GPa})$ of $\mathrm{Li}_{2} \mathrm{CaGeO}_{4}$.

\begin{tabular}{ccccccc}
\hline Compound & $\mathrm{C}_{11}$ & $\mathrm{C}_{12}$ & $\mathrm{C}_{13}$ & $\mathrm{C}_{33}$ & $\mathrm{C}_{44}$ & $\mathrm{C}_{66}$ \\
& & & & & & \\
\hline $\mathrm{Li}_{2} \mathrm{CaGeO}_{4}$ & 196.866 & 52.170 & 61.427 & 78.59 & 60.867 & 54.142 \\
& & & & 8 & & \\
\hline $\mathrm{CuMn}_{2} \mathrm{InSe}_{4}[26]$ & 102.8 & 72.7 & 58.8 & 70.5 & 18.3 & 21.7 \\
\hline
\end{tabular}

Finally, the bulk modulus $B$ and shear modulus $G$, based on Hill approximation are expressed as:

$$
B_{H}=\frac{1}{2}\left(B_{v}+B_{R}\right)
$$

and

$$
G_{H}=\frac{1}{2}\left(G_{v}+G_{R}\right)
$$

The values of Young's modulus $E$ and Poisson's ratio $v$ were also calculated by using the equations :

$$
E=\frac{9 G B}{3 B+G}
$$




$$
v=\frac{3 B-2 G}{2(3 B+G)}
$$

Table 3 : The calculated polycrystalline bulk $\left(B_{V}, B_{R}, B_{H}\right.$ in $\left.G P a\right)$, shear $\left(G v, G_{R}, G_{H}\right.$ in $\mathrm{GPa}$ ), and Young's (E in GPa) modulus, Poisson's $\sigma$ ratios and compressibility $\beta$ of $\mathrm{Li}_{2} \mathrm{CaGeO}_{4}$ compound.

\begin{tabular}{|l|l|l|l|l|l|l|l|l|l|}
\hline Compounds & $\mathrm{B}_{\mathrm{V}}$ & $\mathrm{B}_{\mathrm{R}}$ & $\mathrm{B}_{\mathrm{H}}$ & $\mathrm{G}_{\mathrm{V}}$ & $\mathrm{G}_{\mathrm{R}}$ & $\mathrm{G}_{\mathrm{H}}$ & $\mathrm{E}$ & $\sigma$ & $B$ \\
\hline $\mathrm{Li}_{2} \mathrm{CaGeO}_{4}$ & 91.37 & 74.92 & 83.15 & 54.10 & 45.15 & 50.07 & 148.74 & 0.247 & 0.013 \\
& & & & & & & & & \\
\hline $\mathrm{CuMn}_{2} \mathrm{InSe}_{4}[23]$ & 70.72 & - & - & 16.6 & - & - & - & 0.3895 & - \\
\hline
\end{tabular}

The evaluated values of the bulk modulus $B$, shear modulus $G$, Young's modulus $E$ and Poisson's ratio $\sigma$ of $\mathrm{Li}_{2} \mathrm{CaGeO}_{4}$ using the VRH approximations are given in Table 3. The bulk and shear modulus can be explained as a measure of the resistance to volume and shape changes respectively. As can be seen from Table 3, that the low values of $B$ and $G$ suggest that the $\mathrm{Li}_{2} \mathrm{CaGeO}_{4}$ should be soft materials that can be easily machinable. The Young's modulus E assesses the stiffness of the compound: the larger the value of Young's modulus the stiffer will be the compound. On comparing the results on $G$ with those reported for $\mathrm{CuMn}_{2} \mathrm{InSe}_{4}[28]$. The Young's modulus of $\mathrm{Li}_{2} \mathrm{CaGeO}_{4}$ is slightly larger than that of $\mathrm{CuMn}_{2} \mathrm{InSe}_{4}$ which means that the former is a much harder than the latter. The studied compound has a moderate bulk modulus, indicating that its resistant to compression is moderate, i.e., it has a high compressibility.

The calculated 3D representations of the directional dependence of the Young's modulus $(\mathrm{E})$ and compressibility $(\beta)$ and their cross sections in the $\mathrm{xy}$ and $\mathrm{xz}$ planes are shown in Fig. 2. Obviously, the 3D-closed surfaces (cross sections) of $E$ and $\beta$ exhibit a pronounced deviation from the spherical shape (circular form), indicating a noticeable directional dependence of the Young modulus and compressibility. Thus, one concludes that $\mathrm{Li}_{2} \mathrm{CaGeO}_{4}$ is characterized by a pronounced elastic anisotropy. 
The investigation of the electronic band structure and total density of states are important because most of the physical properties of solids are related to them. The spinpolarized band structure in its tetragonal structure has been calculated using the FP-LAPW method. Based on the lattice symmetry, the integration paths $\mathrm{P}(0,0,0.5)-\Gamma(0,0,0)-\mathrm{N}(0.5$, $0.5,0.5)-\mathrm{H}(0,0.5,0.5)-\Gamma(0,0,0)$ are performed so as to treat the band structure for tetragonal phase. The obtained results are plotted in Figs. 3 and 4. The energy gap obtained from FP-LPAW approach is $4.49 \mathrm{eV}$, while that obtained from PP-PW calculations is 4.14 $\mathrm{eV}$. For thus compound, the DOS is basically divided into three parts, at lower energy core electrons where we find the contribution of $\mathrm{Ca}$ s and $p$ states and small contribution Ge $\mathrm{s}$; the valance band is formed by Ca-p, Ge-s and Ge-p bonding orbitals but the Ca- 4 give large contribution compared to Ge-s and Ge-p. In the conduction band, the Li-p, Li-s, Ge-s and Ge-p states are contributing. Here we see that the main contributions are due to Li-p and $\mathrm{Li}-\mathrm{s}$ and the minor contributions are due to Ge-s and Ge-p orbitals.

Meanwhile, the electronic structure has also been computed versus pressure and plotted in Fig. 5. We observe a clear shift of the Fermi level. Nevertheless, one can note a preserved semiconducting nature for both band structures of interest in the stress range of $-14 \%$ up to $14 \%$.

The degree of polarization of a material when it is under an external electric field is described by the real part of the dielectric function is described by the real part of the dielectric function. The imaginary part of the dielectric function shows the amount of absorption inside the material under load. It contributes into both intra-band and inter-band transitions. Nevertheless, the intra-band transition contribution is only significant for metals. Besides, the inter-band transition can be further split into direct and indirect transitions. The indirect inter-band transitions that involve phonons scattering are expected to have only a little contribution to the imaginary part of the dielectric function and hence are neglected. In Fig 6, we present the dielectric function of $\mathrm{Li}_{2} \mathrm{CaGeO}_{4}$ as calculated by FP-LAPW and PP-PW methods. Note that the values of the dielectric function of the material of interest are large within near (3.10-4.13 eV) and middle (4.13-6.20 eV) ultraviolet regions but become smaller in the far (6.20-12.4 eV) ultraviolet region. Our analysis of the $\varepsilon_{2}(\omega)$ spectra shows that the threshold energy (first critical point) of the dielectric function occurs at about $4.81 \mathrm{eV}$ and $4.23 \mathrm{eV}$ for FP-LAPW and PP-PW methods, respectively. These points are mainly coming from the electron transition from 
the $\mathrm{Ca} \mathrm{p}(\mathrm{VB})$ to $\mathrm{Ge} \mathrm{s}(\mathrm{CB})$ orbitals. We note that in $\varepsilon_{2}(\omega)$ shows a peak located at $6.81 \mathrm{eV}$ and $6.17 \mathrm{eV}$ for FP-LAPW and PP-PW methods, respectively. This point is mainly derived from the transition from Ge $\mathrm{p}(\mathrm{VB})$ to Ge $\mathrm{s}(\mathrm{CB})$ orbitals. In additional, the peak is located at $7.23 \mathrm{eV}$ for FP-LAPW method and $7.03 \mathrm{eV}$ for PP-PW method. This point is mainly derived from the transition from Ge $\mathrm{p}(\mathrm{VB})$ to $\mathrm{Li} \mathrm{p}(\mathrm{CB})$ orbitals. The behaviour of $\varepsilon_{1}(\omega)$ seems to be rather similar to that of $\varepsilon_{2}(\omega)$. Below the reststrahlen region in the optical spectra, the real part of the dielectric function asymptotically approaches the static or lowfrequency dielectric constant $\varepsilon_{1}^{x x}(0)$ and $\varepsilon_{1}^{z z}(0)$. In the present contribution, the calculated static dielectric constant $\varepsilon_{1}^{x x}\left(\varepsilon_{1}^{z z}(0)\right)$ is found to be about 3.27(3.33) and 2.54(2.32) using FP-LAPW and PP-PW methods, respectively.

The refractive index is a quantity that describes how much light is refracted after entering a material [29]. Fig7, illustrate the refractive index $n(\omega)$ and the extinction coefficient $K(\omega)$ of $\mathrm{Li}_{2} \mathrm{CaGeO}_{4}$ material. From the refractive index plot, we remark that the material possesses high refractive index within visible and Middle Ultraviolet and decreases at higher energy in the Far ultraviolet $(6.20-10.60 \mathrm{eV})$. In addition, the static refractive indexes $n^{x x}\left(\varepsilon^{z z}(0)\right)$ for FP-LAPW and PP-PW are 1.80(1.82) and 1.59(1.52), respectively. Hence, $\mathrm{Li}_{2} \mathrm{CaGeO}_{4}$ compound can be used for devices such as Bragg's reflectors, optical and optoelectronic devices.

\section{CONCLUSION}

In conclusion, the structural, electronic, elastic and optical properties of tetragonal $\mathrm{Li}_{2} \mathrm{CaGeO}_{4}$ were investigated using both PP-PW and FP-LAPW methods. The computed lattice parameters, namely $a$ and $c$ and the bulk modulus and its first pressure derivative were found to be in good accord with data available in the literature. Band structure and densities of states (DOS) of $\mathrm{Li}_{2} \mathrm{CaGeO}_{4}$ have been obtained. Calculations show that thus materials posses indirect energy band gap $(\mathrm{H} \rightarrow \Gamma)$ of about $4.49 \mathrm{eV}$ for FP-LAPW and 4.14 eV for PP-PW methods. The elastic constants, bulk modulus, shear modulus, Young's modulus, Poisson's ratio and compressibility are systematically presented.Optical properties reveal that these compound are suitable candidate for optoelectronic devices in the visible and ultraviolet (UV) regions. The semiconducting materials $\mathrm{Li}_{2} \mathrm{CaGeO}_{4}$ of interest were found to be stable against volume change of 0 to $+14 \%$. 


\section{References}

[1] G. M. Ford, Q. Guo, R. Agrawal, H. W. Hillhouse, W. Hugh, Chem. Mater. 232626 (2011).

[2] Q. Guo, G. M. Ford, W. C. Yang, B. C. Walker, E. A. Stach, H. W Hillhouse and R. Agrawal, J. Amer. Chem. Soc. 13217384 (2010).

[3] S. Berri, J. Supercond. Novel Magn. 311941 (2018).

[4] G.C. Catella, D. Burlage, MRS Bull. 2328 (1998).

[5] P. Grima-Gallardo, K. Cárdenas, L. Molina, M. Quintero, J. Ruiz, G. E. Delgado, J.M. Briceño,Phy. Stat. Sol. (a) 187395 (2001).

[6] G.H. McCabe, T. Fries, M.T. Liu, Y. Shapira, L.R. Ram-Mohan, R. Kershaw, A. Wold, C. Fau, M. Averous, E.J. McKnight, Phys. Rev. B 566673 (1997).

[7] S. Chen, X.G. Gong, A. Walsh, S.H. Wei, Phys. Rev. B 79165211 (2009).

[8] Y.G. Davydyuk, V.P. Sachanyuk, S.V. Voronyuk, I.D. Olekseyuk, Y.E. Romanyuk, O.V. Parasyuk : Physica B 373(2) 355 (2006).

[9] Davydyuk, G.E., Myronchuk, G.L., Kityk, I.V., Danyl'chuk, S.P., Bozhko, V.V., Parasyuk, O.V.: Opt. Mater. 33(8) 1302 (2011).

[10] V S Vavilov : Phys.-Usp. 37269 (1994)

[11] H. Hamad, C. Raynaud, P. Bevilacqua, D. Tournier, B. Vergne, D. Planson, "Optical Beam Induced Current Measurements based on two-photon absorption process in $4 \mathrm{H}-\mathrm{SiC}$ bipolar diodes", Applied Physics Letters 104082102 (2014).

[12] J.A.Gard and A.R.West, Journal of Solid State Chemistry 7(4) 422 (1973).

[13] P. Blaha, K. Schwarz, G. K. H. Madsen, D. Kvasnicka, J. Luitz, WIEN2K, an Augmented Plane Wave +Local orbitals program for calculating crystal properties (Karlheinz Schwarz, Technische Universität, Wien, Austria, 2001), ISBN 3-9501031-1-2.

[14] J.P. Perdew, S. Burke, M. Ernzerhof, Phys. Rev. Lett., 773865 (1996).

[15] D. Vanderbilt, Phys. Rev. B, 417892 (1990).

[16]J.P. Perdew, A. Ruzsinszky, G.I. Csonka, O.A. Vydrov, G.E. Scuseria, L.A. Constanti n, X. Zhou and K. Burke, Phys. Rev. Lett 100136406 (2008).

[17] M.D. Segall, P.J.D. Lindan, M.J. Probert, C.J. Pickard, P. J. Hasnip, S. J. Clark, M.C.

Payne, J. Phys.: Condens. Matter 142717 (2002). 
[18] T. H. Fischer, J. Almlof, J. Phys. Chem. 969768 (1992).

[19] A.H. Reshak, S. Auluck, Phys Rev B. 68245113 (2003).

[20] A. Delin, A. O. Eriksson, R. Ahuja, B. Johansson, M. S. Brooks, T. Gasche, S. Auluck and J. M. Wills Phys. Rev. B 541673 (1996).

[21] F.D. Murnaghan, Proc. Natl. Acad. Sci. U. S. A. 30244 (1944).

[22] F. Birch, Phys. Rev. 71809 (1974).

[23]Yu, R., Zhu, J., Ye, H.Q.: Computer Physics Communications. 181671 (2010).

[24]Born, M., Huang, K.: Dynamics theory of crystal lattices. Oxford University Press, Oxford (1954).

[25] R. Hill, Proc. Phys. Soc., London, Sect. A 65349 (1952).

[26] W. Voigt, Lehrbuch de Kristallphysik, Terubner, Leipzig, (1928).

[27] A. Reuss, Z. Angew. Math. Mech. 949 (1929).

[28] L. Salik, A. Bouhemadou, K. Boudiaf, et al. J Supercond Nov Magn 331091 (2020).

[29] R. Saniz, Lin-Hui Ye, T. Shishidou, A.J.Freeman Phys. Rev. B, 74014209 (2006).

\section{Figure caption}

Figure. 1: Normalized energy as a function of volume for $\mathrm{Li}_{2} \mathrm{CaGeO}_{4}$.

Figure 2 : The directional dependence of the Young's modulus and compressibility as well as their cross sections in the xy (001) and xz planes (010) crystallographic planes.

Figure.3 : Band structure for high-symmetry directions in the Brillouin zone using FPLAPW method.

Figure. 4 Total and projected density of states for $\mathrm{Li}_{2} \mathrm{CaGeO}_{4}$ using FP-LAPW method.

Figure 5: Band structure under pressure effect.

Figure.6 The real part $\varepsilon_{1}(\omega)$ and imaginary part $\varepsilon_{2}(\omega)$ of dielectric constant $\varepsilon(\omega)$.

Figure.7 Calculated refractive index $n(w)$ and extinction coefficient $k(w)$ 


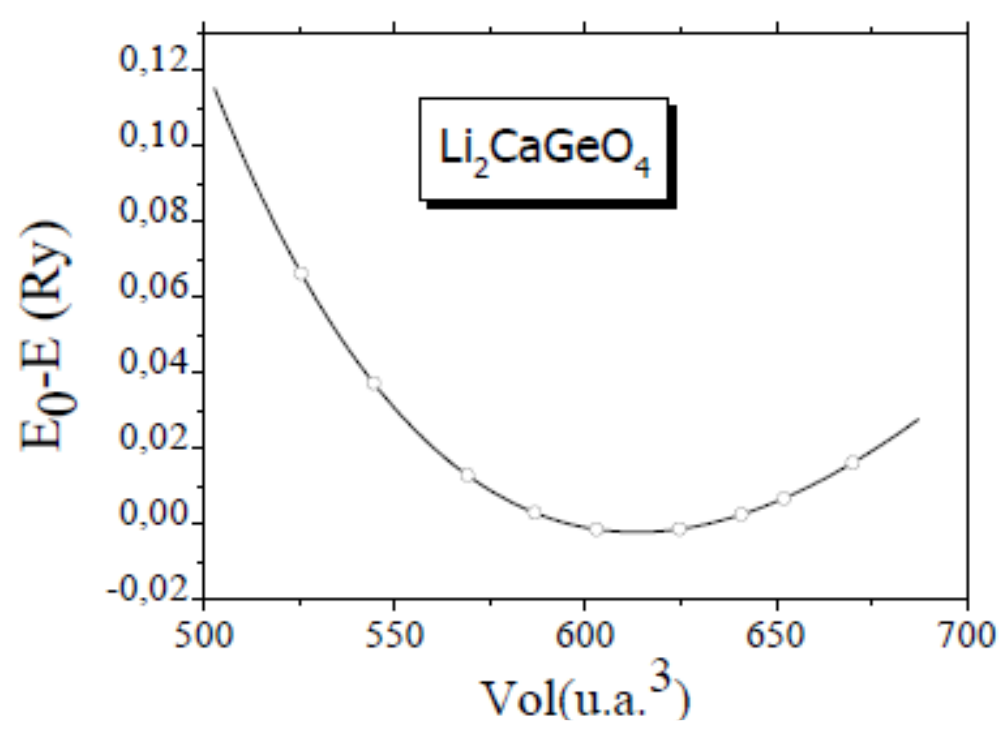

Young's modulus

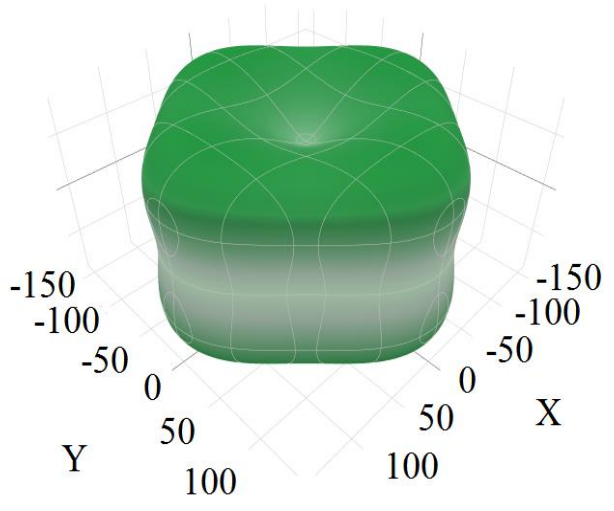

Compressibility

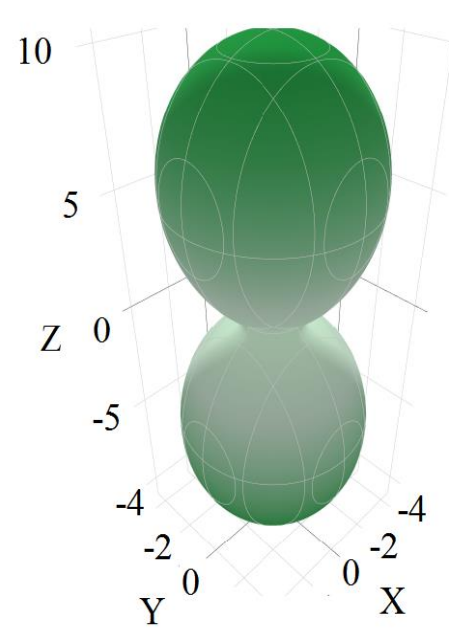




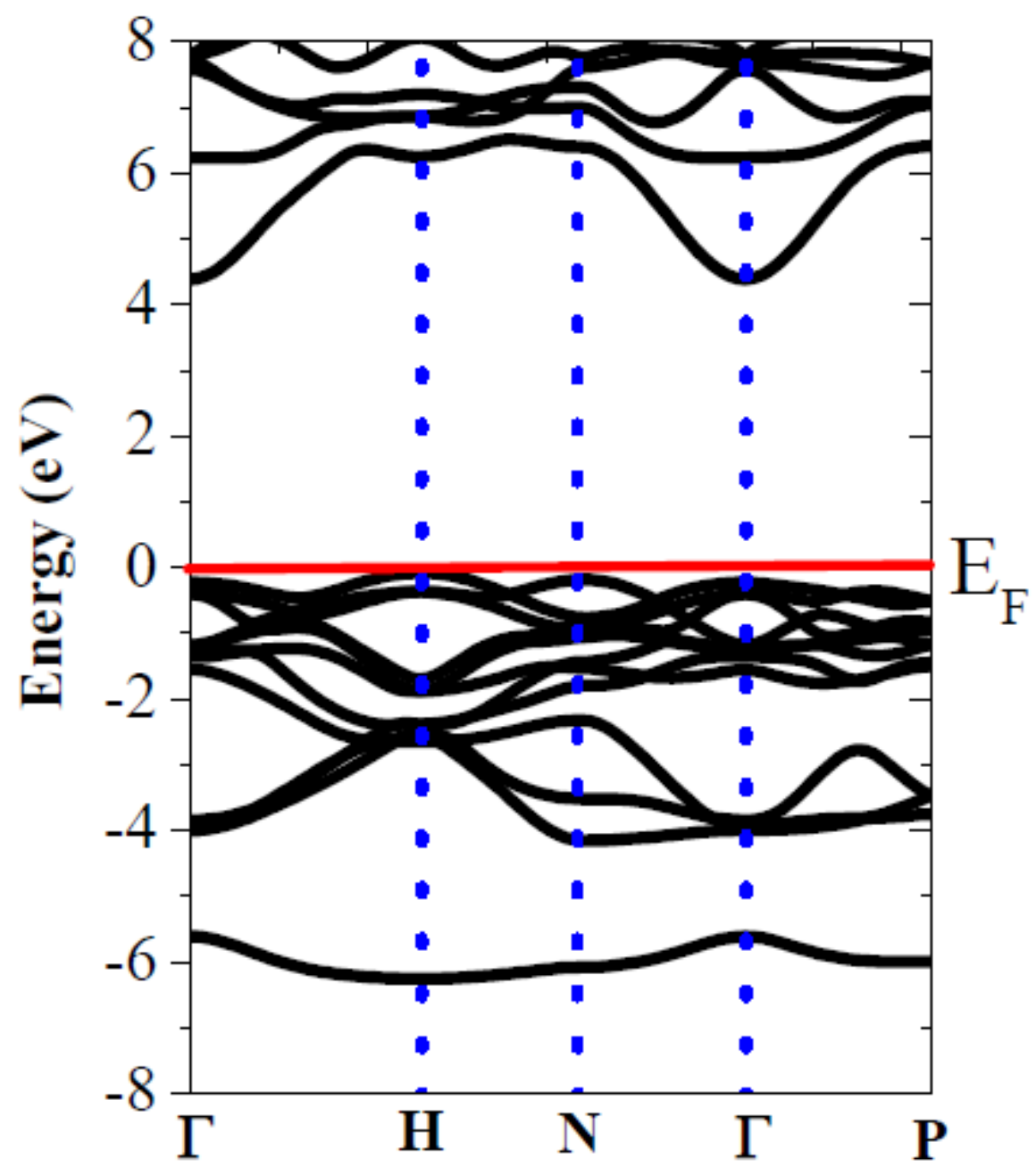




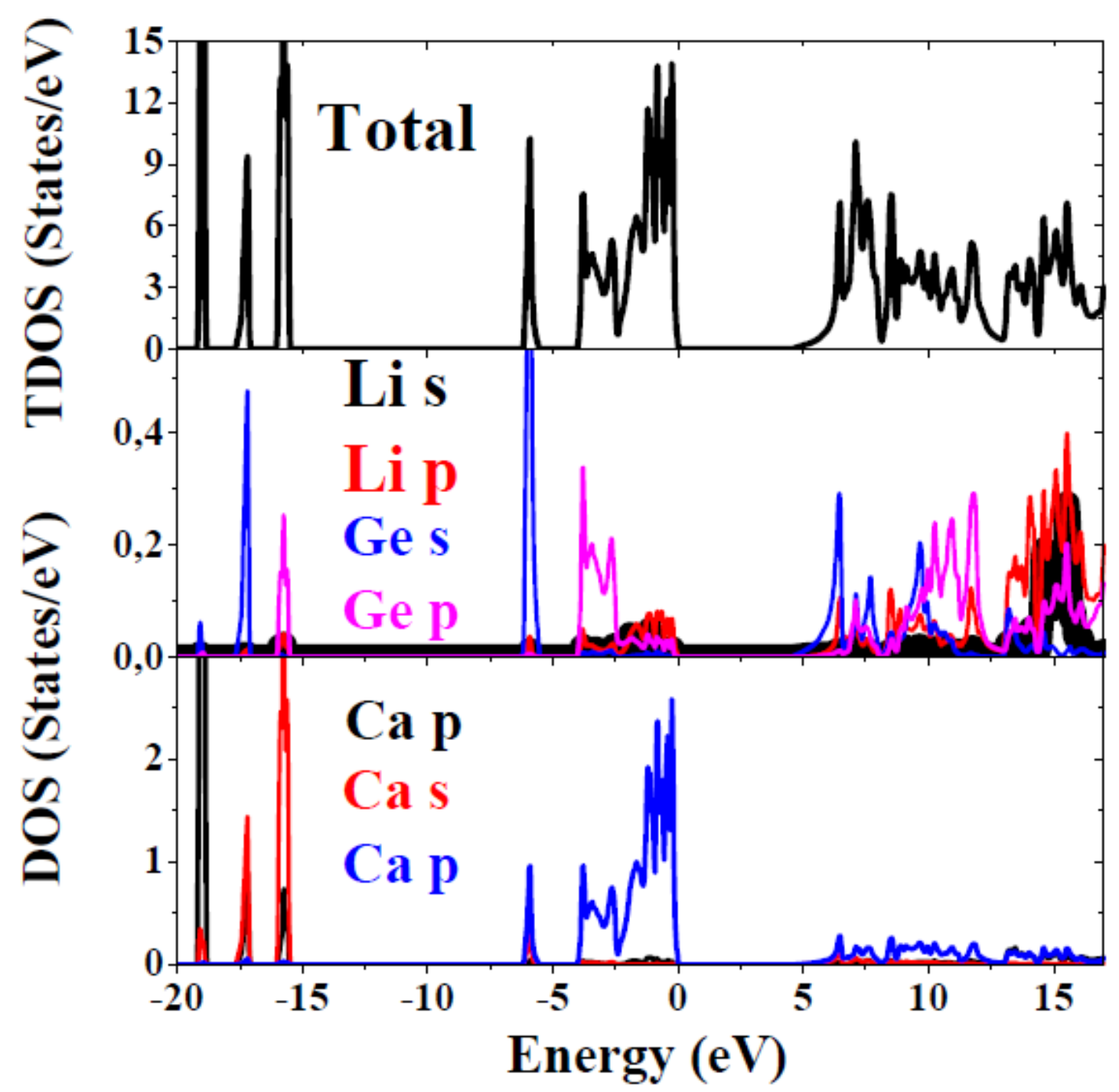



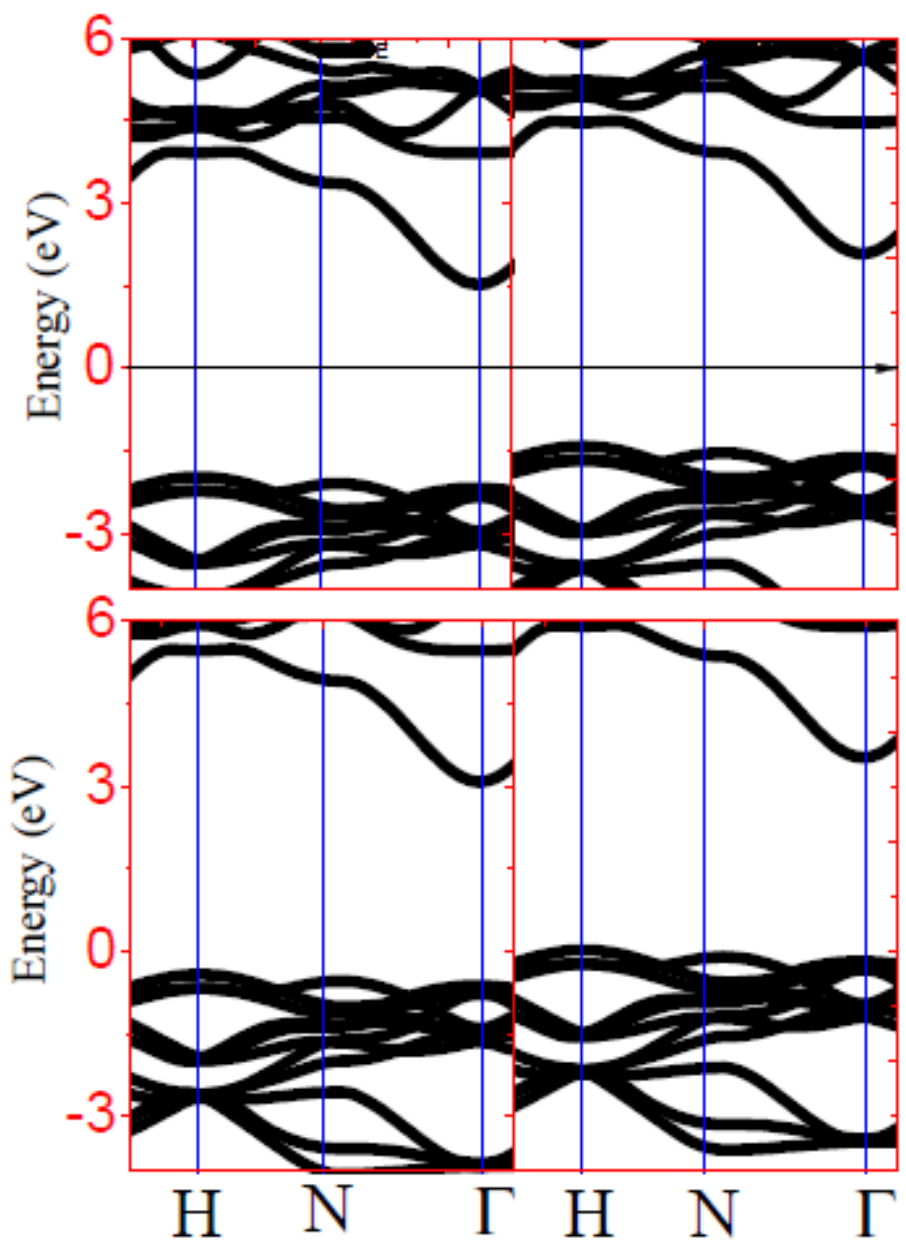

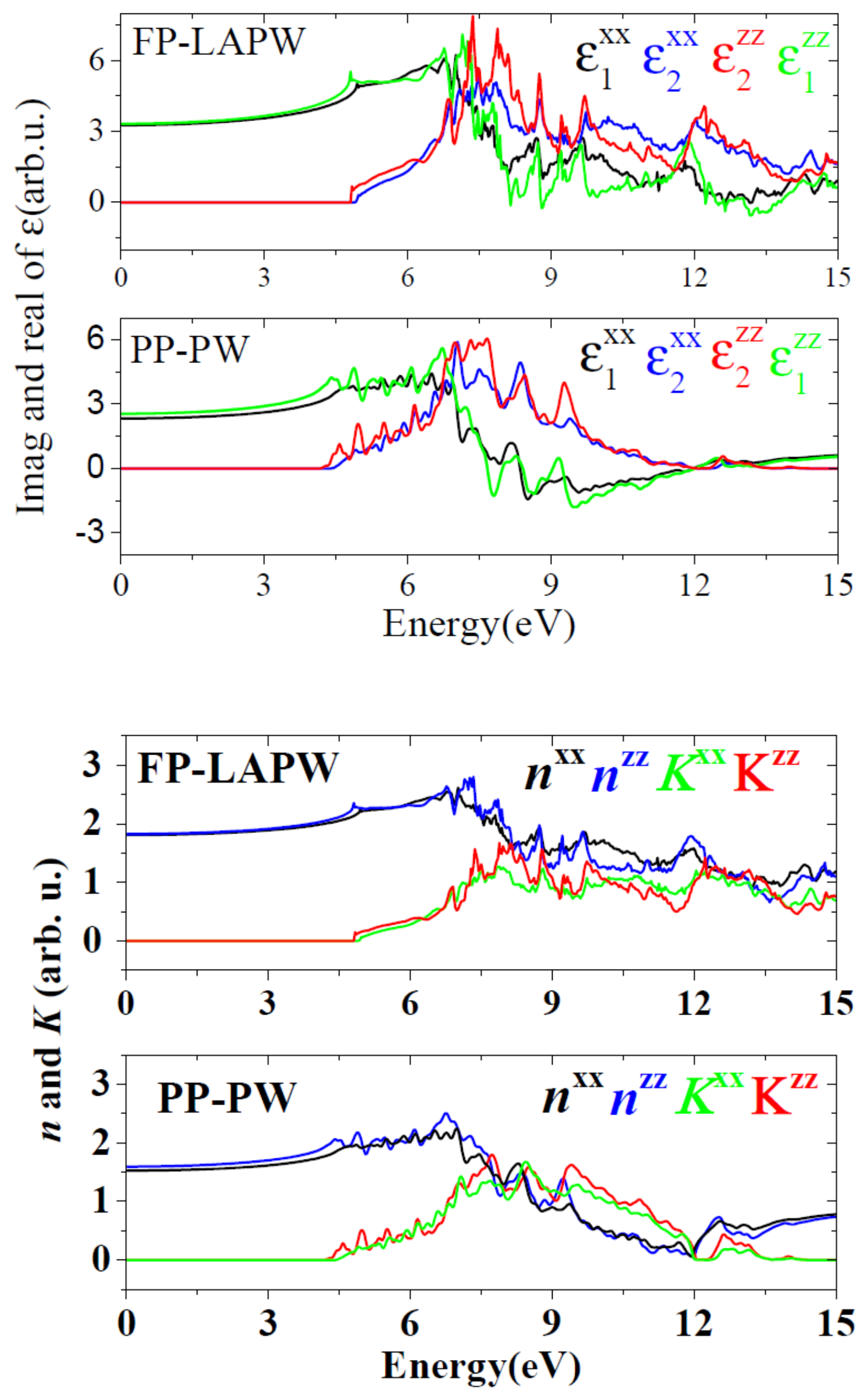